\title{
Dry adhesive bonding of nanoporous inorganic membranes to microfluidic devices using the $\operatorname{OSTE}(+)$ dual-cure polymer
}

\author{
Farizah Saharil $^{1}$, Fredrik Forsberg ${ }^{1}$, Yitong Liu ${ }^{1}$, \\ Paolo Bettotti ${ }^{2}$, Neeraj Kumar ${ }^{2}$, Frank Niklaus ${ }^{1}$, \\ Tommy Haraldsson ${ }^{1}$, Wouter van der Wijngaart ${ }^{1}$, \\ and Kristinn B Gylfason ${ }^{1}$ \\ 1 Microsystem Technology Laboratory, School of Electrical Engineering, \\ KTH Royal Institute of Technology, 10044 Stockholm, Sweden \\ 2 Nanoscience Laboratory, Department of Physics, \\ University of Trento, 38123 Povo (TN), Italy \\ E-mail: kristinn.gylfason@ee.kth.se
}

\begin{abstract}
We present two transfer bonding schemes for incorporating fragile nanoporous inorganic membranes into microdevices. Such membranes are finding increasing use in microfluidics, due to their precisely controllable nanostructure. Both schemes rely on a novel dual-cure dry adhesive bonding method, enabled by a new polymer formulation: $\operatorname{OSTE}(+)$, which can form bonds at room temperature. $\operatorname{OSTE}(+)$ is a novel dual-cure ternary monomer system containing epoxy. After the first cure, the $\operatorname{OSTE}(+)$ is soft and suitable for bonding, while during the second cure it stiffens and obtains a Young's modulus of $1.2 \mathrm{GPa}$. The ability of the epoxy to react with almost any dry surface provides a very versatile fabrication method. We demonstrate the transfer bonding of porous silicon and porous alumina membranes to polymeric microfluidic chips molded into OSTE $(+)$, and of porous alumina membranes to microstructured silicon wafers, by using the OSTE $(+)$ as a thin bonding layer. We discuss the $\operatorname{OSTE}(+)$ dual-cure mechanism, describe the device fabrication, and evaluate the bond strength and membrane flow properties after bonding. The membranes bonded to OSTE $(+)$ chips delaminate at $520 \mathrm{kPa}$, and the membranes bonded to silicon delaminate at $750 \mathrm{kPa}$, well above typical maximum pressures applied to microfluidic circuits. Furthermore, no change in membrane flow resistance was observed after bonding.
\end{abstract}




\section{Introduction}

Recent advances in bottom-up lithography-free nanofabrication have made it possible to pattern nanoscale features over wafer scale areas with good homogeneity. A good example is the electrochemical etching of nanoporous membranes into inorganic materials such as silicon and alumina $[1,2]$. The electrochemical processes employed enable tailored pore sizes in the range from nano- to micrometers, and such nanoporous membranes are now finding numerous applications in chemistry, biology and medicine, due to their ability to discriminate molecules based on size and interaction. Currently, they are used extensively in filtering [1], amplification [3], sequencing [4], and drug delivery [5]. Furthermore, these membranes provide a large internal surface, making them attractive as binding surfaces for biosensing $[6,7]$.

However, to create functional microdevices, lithographic top-down structuring of e.g. mixers, multiplexers, valves and so forth is required. These functions are usually patterned in plastics, glass or silicon, using processes not compatible with the electrochemical bottom-up structuring. The incompatibility stems both from the extreme requirements on substrate uniformity, to maintain a uniform electric field during electrochemical etching, and from the corrosive chemical employed. Here, we present a transfer bonding method that enables the heterogeneous integration of top-down and bottom-up structured parts fabricated separately under optimal conditions. This way, heterogeneous devices can be created that combine nano- and microfluidic functions in novel ways, without compromising performance.

Examples of reported heterogeneous integration of nanoporous membranes include the transfer and patterning of porous silicon (por-Si) bragg mirrors from a silicon source wafer to a flexible polymer sheet using a polydimethylsiloxane (PDMS) stamp [8], and the transfer and wet adhesive polymer bonding of a por-Si waveguide on glass [9]. Heterogeneous integration by transfer bonding has recently been extensively reviewed in [10]. A few approaches to the integration of nanoporous inorganic membranes into microfluidic devices have been reported. At one end of the spectrum are monolithic techniques, e.g. in-situ forming of porous silicon membranes in silicon microchannels [11]. However, as mentioned above, monolithic integration limits the choice of manufacturing processes, due to compatibility requirements. At the other end of the spectrum is post fabrication integration by clamping or gluing $[12,13]$. These methods maintain full processing freedom, and membranes with particular properties can even be ordered from specialized suppliers. However, due to the extreme fragility of thin porous membranes, clamping is a low yield process, and the capillary action of porous membranes makes them prone to clogging when using liquid glue $[14,15]$. The integration of membranes into microfluidic devices was reviewed in [16]. Dry adhesive bonding of the membranes directly to the microfluidic device elegantly solves these problems, but no simple and generic method was previously available. Here, we present such a method, enabled by the dry polymer adhesive $\operatorname{OSTE}(+)$.

We have recently introduced a family of off-stoichiometry thiol-ene (OSTE) 
polymers, developed specifically to bridge the gap between research prototyping and commercialization of lab-on-chip devices [17]. Like PDMS, OSTE polymers are compatible with soft lithography, and thus cleanroom access is not necessary for OSTE based fabrication. In contrast to PDMS, however, the OSTE polymers feature tunable mechanical properties, and a large number of surface anchored thiol groups that are capable of participating in "click" reactions [18], allowing easy one-step surface chemistry modifications. Furthermore, OSTE polymers are surface and bulk patternable using UV-light, and designed to soften when heated above their glass transition temperature $\left(T_{\mathrm{g}}\right)$, to conform with micro-irregularities on bond surfaces when pressure is applied. We have previously reported the utility of OSTE for sensor packaging, by the formation of a spontaneous leakage free bond between an OSTE microfluidic structure and the gold surface of a quartz crystal microbalance (QCM) [19], and for "biostickers", where OSTE microfluidic structures are spontaneously bonded under biocompatible conditions to spotted protein and DNA microarray surfaces [20].

Here, we introduce a novel dual-cure off-stoichiometry thiol-ene-epoxy $(\operatorname{OSTE}(+))$ polymer material, as an extension to the OSTE family. By adding epoxy to the OSTE formulation, it gains the ability to directly react with almost any dry surface, and the two stage curing facilitates bonding, by providing a compliant bond surface after the first stage cure that is subsequently hardened fully upon the second cure. Furthermore, since no monomers remain unreacted after the second cure, no leachable compounds that can hamper bond integrity remain in the bond area. We discuss the properties of the $\operatorname{OSTE}(+)$ material in more detail in section 2 below.

In this work, we describe the use of $\operatorname{OSTE}(+)$ for incorporating nanoporous inorganic membranes into microfluidic devices on either the chip- or the waferlevel. In section 3, we describe a method for integrating both por-Si membranes, and commercially available porous alumina (por-Alu) membranes, into an OSTE $(+)$ polymeric chip, using the OSTE $(+)$ photopatterning capability to make vias. This is an extension of our previous work [21], where through-holes were made by drilling. In section 4, we describe a method for wafer-scale integration of the por-Alu membranes onto a full silicon wafer, using a thin $\operatorname{OSTE}(+)$ bonding layer. Finally, in section 5 we verify that the porous membranes maintain their flow characteristics after bonding, and characterize the bond strength of both methods by applying pressure to the bond interface.

\section{2. $\operatorname{OSTE}(+)$ material properties}

The OSTE $(+)$ polymer formulation consists of thiol (Pentaerythritol tetrakis (2mercaptoacetate)), allyl (the -ene of the formulation, in this case triallyl-1,3,5-triazine2,4,6(1H,3H,5H)-trione), and epoxy (Bisphenol A Diglycidyl Ether, BADGE) monomers (all acquired from Sigma-Aldrich). The stoichiometric ratios of the OSTE $(+)$ used here were 1.5:1.0:0.5, for thiol, allyl, and epoxy, respectively.

In the first step of the dual-cure process, the thiol and allyl monomers react at 


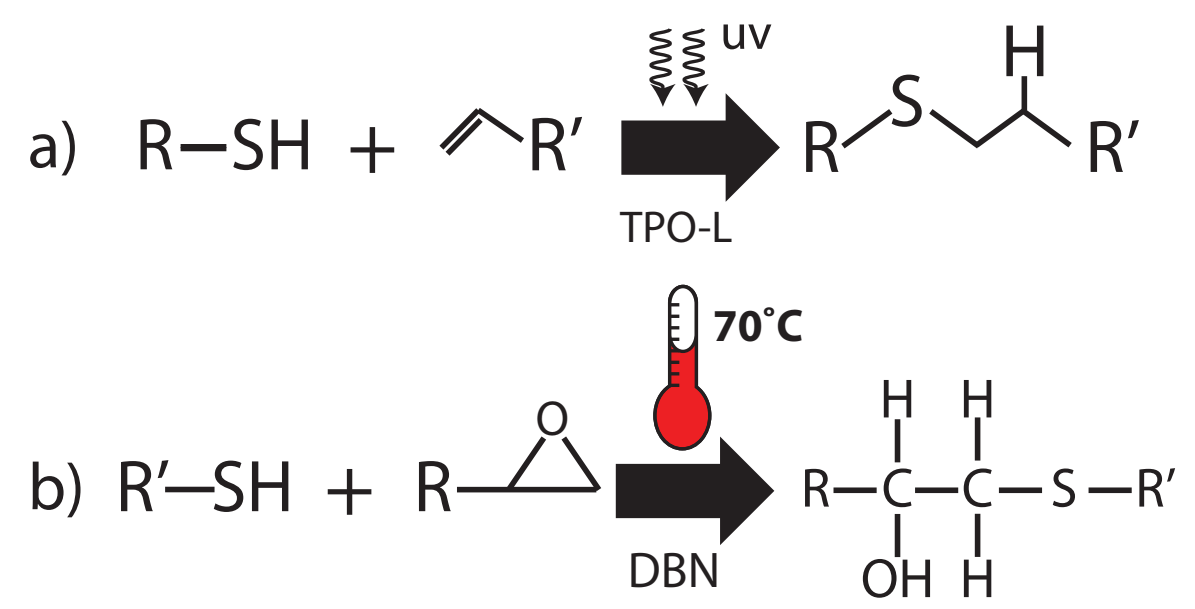

Figure 1. The reaction mechanism of the dual-cure ternary monomer system OSTE(+): a) In the first stage UV cure, half the thiol reacts with the allyl, initiated by TPO-L. b) In the second stage cure, the remaining thiol reacts with epoxy, initiated by DBN. The reaction is accelerated by heating to $70{ }^{\circ} \mathrm{C}$ after bonding.

room temperature, via an alternating radical copolymerization initiated by Lucirin TPO-L (Ethyl-2,4,6-Trimethylbenzoylphenylphosphinate, acquired from BASF AG, Ludwigshafen, Germany) and UV-light (Figure 1 a). In this step, all allyl and half the thiols are consumed, resulting in a partially polymerized soft solid material that can be handled. This intermediate product is ideal for direct dry bonding to many kinds of substrates, such as silicon and glass, since its softness makes it compliant to irregularities on the substrate, and since it is able to form covalent epoxy and thiol links upon further cure.

In the second cure, the thiol and epoxy react, via an alternating anionic mechanism initiated by DBN (1,5- diazabicyclo[4.3.0]non-5-ene from Sigma-Aldrich), as shown in Figure $1 \mathrm{~b}$. The reaction starts upon mixing, but proceeds slowly at room temperature (full curing in 1-2 days). To accelerate the reaction after bonding, we raise the temperature to $70{ }^{\circ} \mathrm{C}$ for $1-2 \mathrm{~h}$. The second cure affords covalent bonding to rigid substrates and yields materials with $T_{\mathrm{g}}$ above $70^{\circ} \mathrm{C}$ and a Young's modulus of $1.2 \mathrm{GPa}$. We have analyzed the $\operatorname{OSTE}(+)$ reaction mechanism in more detail in [21].

\section{Transfer bonding of porous membranes to $\operatorname{OSTE}(+)$ polymer chips}

In this section, we demonstrate the transfer bonding of porous membranes onto polymeric OSTE $(+)$ chips. We demonstrate both the fabrication and transfer bonding of por-Si membranes that we make ourselves (por-Si/OSTE $(+)$ ), and the transfer bonding of commercially available por-Alu membranes (por-Alu/OSTE $(+)$ ).

The fabrication flow for the por-Si membrane integration is illustrated in Figure 2. In part a, the por-Si membranes are etched into a (100) n-type $(0.1 \Omega \mathrm{cm})$ silicon substrate by an electrochemical process. In the first step, the membranes were defined 

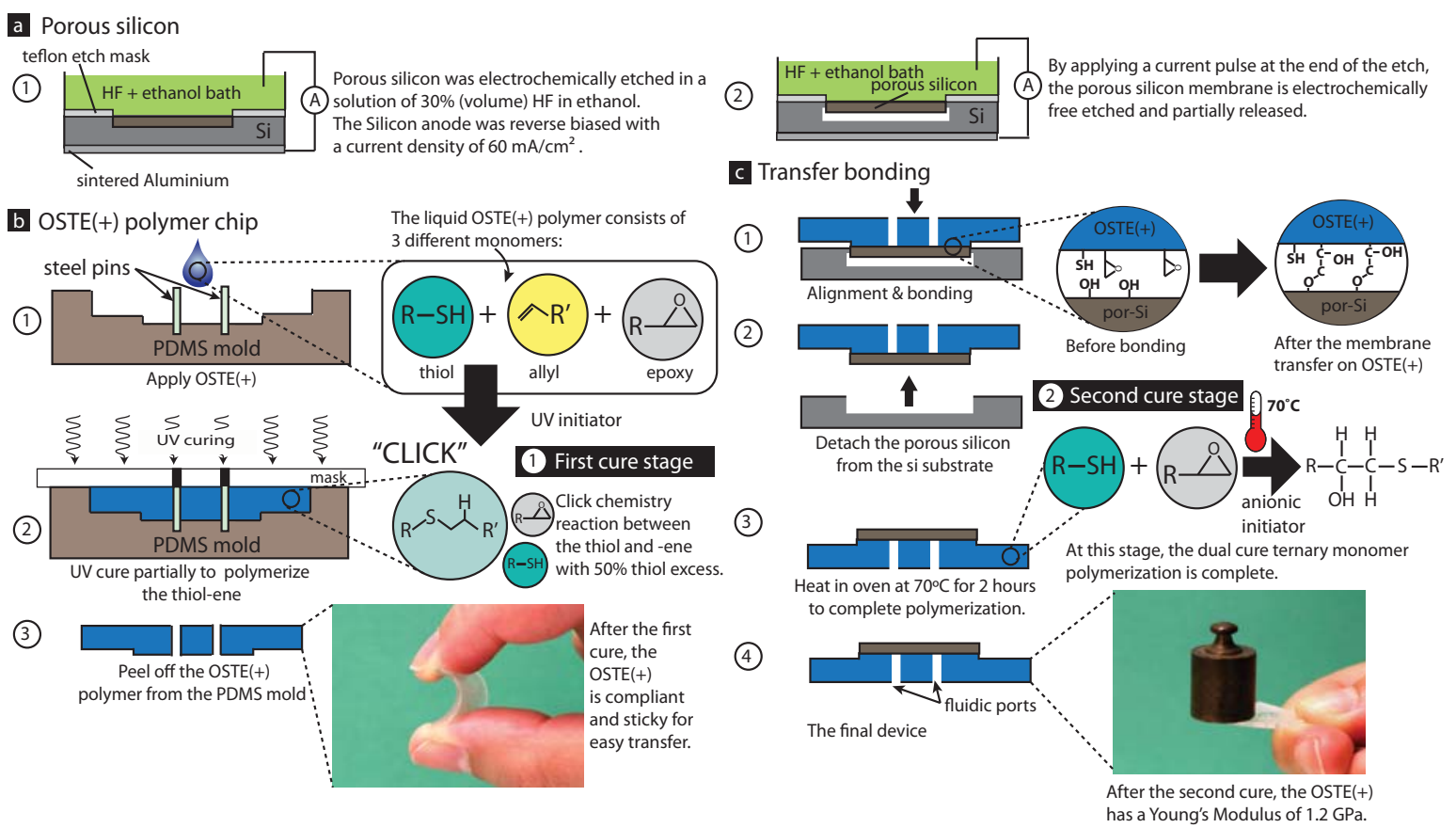

Figure 2. The fabrication sequence of $\operatorname{OSTE}(+)$ polymer chips with dry bonded porous silicon membranes: a) The porous silicon etching. b) Casting of the OSTE $(+)$ chip, by the first curing step. c) Dry transfer bonding of the porous silicon membrane onto the $\operatorname{OSTE}(+)$ chip, and second stage cure.

by etching in a solution of $30 \%$ (by volume) hydrofluoric acid (HF) in ethanol, using a Teflon mask to limit the etching to a disk of $6 \mathrm{~mm}$ diameter. The silicon anode was reverse-biased with a current density of $60 \mathrm{~mA} / \mathrm{cm}^{2}$, yielding membranes of $50 \%$ porosity and $70 \mathrm{~nm}$ pore size. The etching was timed to produce $30 \mu \mathrm{m}$ thick por-Si membranes. By applying a current pulse at the end of the etching process, electropolished is initiated to partially release the por-Si membranes from the Si substrate [22]. The partial release is essential for successful transfer bonding onto the $\operatorname{OSTE}(+)$ chips, since the fragile membranes remain supported by the silicon donor substrate until they are brought in contact with the $\operatorname{OSTE}(+)$ target. Figure 3 a) shows a scanning electron (SEM) image of the cross-section of a por-Si membrane, and Figure $3 \mathrm{~b}$ ) shows a por-Si membrane that has been transferred from its silicon substrate to a plastic carrier.

Figure $2 \mathrm{~b}$ shows the fabrication of the $\operatorname{OSTE}(+)$ chips. As described in section 2, the OSTE(+) prepolymer was prepared with a $50 \%$ thiol excess, $0.1 \%$ of the UV initiator Lucirin TPO-L, and $0.1 \%$ of the anionic initiator DBN. The components were then mixed thoroughly and degassed in a vacuum desiccator, prior to pouring the mixture into mold made of PDMS (step 1). The PDMS mold defines the outer chip dimensions of $15 \times 15 \times 1 \mathrm{~mm}^{3}$, and contains $800 \mu \mathrm{m}$ diameter steel pins that define vias through the chip. To avoid the formation of a residual squeeze-film on top of the pins, a matching shadow mask printed onto a $1 \mathrm{~mm}$ thick polycarbonate (PC) sheet was aligned on top of the mold. The sheet was lightly pressed on top of the uncured OSTE $(+)$ polymer, 


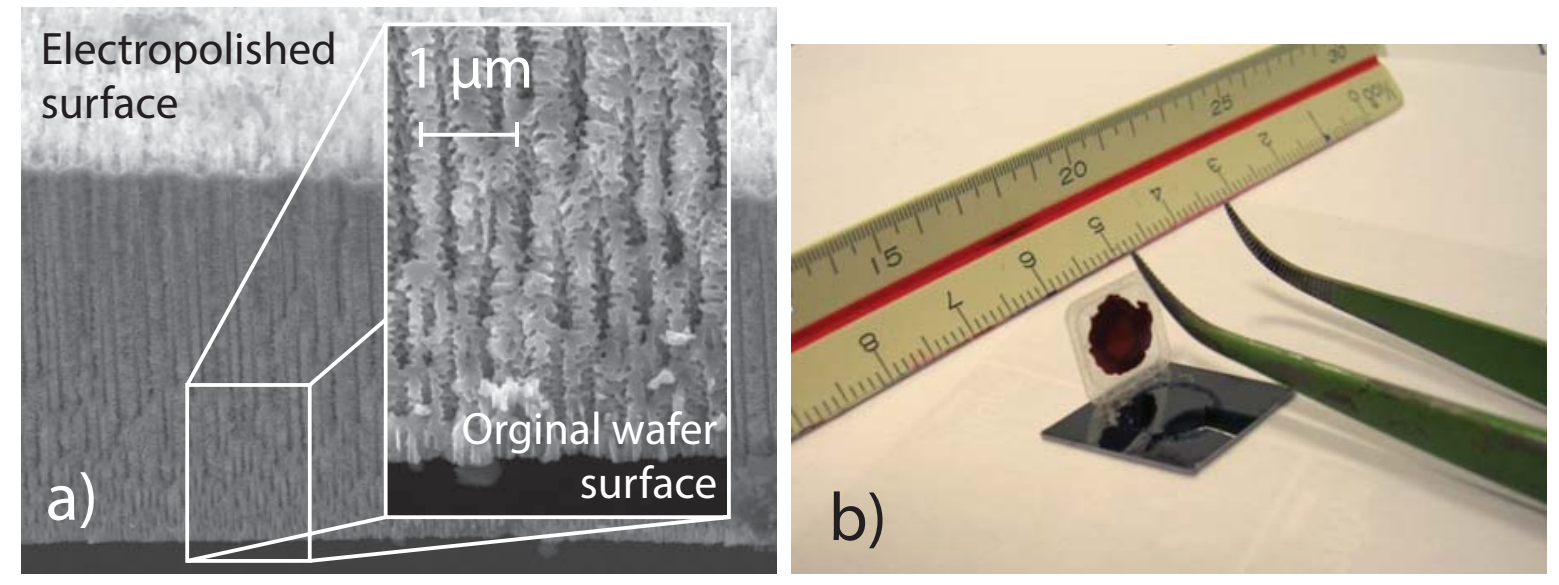

Figure 3. a) An SEM image of a cross-section of a porous silicon membrane. The original wafer surface is at the bottom, and the electropolished interface is at the top. The inset shows an enlarged view of the original silicon surface. b) A photograph of a por-Si membrane that has been transferred from its silicon substrate to a plastic substrate. The ruler scale is in $\mathrm{cm}$.

to ensure a planar top surface (step 2). After the first stage UV curing (90 s at $300 \mathrm{~mW} / \mathrm{cm}^{2}$, using an LS 30/7 1000W UV-lightsource (OAI, USA) with collimating optics and a $365 \mathrm{~nm}$ bandpass filter), the OSTE $(+)$ chip was peeled off from the mold (step 3). At this stage, the surface of the OSTE $(+)$ has unreacted epoxy groups, and has a sticky tape-like property.

The OSTE $(+)$ chip was then aligned and bonded to the por-Si membrane fabricated earlier. Since the membrane was already partially released from the Si substrate by the electropolished step at the end of the electrochemical etch, bringing the sticky $\operatorname{OSTE}(+)$ surface in contact with the membrane yields sufficient adhesion to pull it off the substrate. During the second cure, covalent bonds are formed between the epoxy in the $\operatorname{OSTE}(+)$ and the $\mathrm{OH}$ groups on the por-Si surface. To accelerate the second polymerization, the chip was cured in an oven at $70{ }^{\circ} \mathrm{C}$ for 1 hour. At room temperature, the same reaction takes 1-2 days. After the second cure, the polymeric chip is stiff, as shown in Figure $2 \mathrm{c}$, and serves both as a fluidic circuit and as a mechanical support for the membrane.

The fabrication of the por-Alu/OSTE $(+)$ chips follows the same steps as described in Figure $2 \mathrm{~b}$ and c, except that the por-Alu membranes are delivered from the producer without a supporting substrate. However, since the por-Alu membranes are much more robust than the por-Si membranes, the alumina membranes can be handled individually without the risk of membrane fracture. We used $13 \mathrm{~mm}$ diameter and $60 \mu \mathrm{m}$ thick Whatman Anopore 100 porous alumina membranes (acquired from VWR). According to the supplier's specification, Anopore 100 membranes have a 2 layer structure, with a $2 \mu \mathrm{m}$ thick top layer with a $100 \mathrm{~nm}$ pore size and $40 \%$ porosity, and a $58 \mu \mathrm{m}$ thick bottom layer of $200 \mathrm{~nm}$ pore size and $25 \%$ porosity. Figure 4 a) and b) show photographs of completed por-Si/OSTE(+) and por-Alu/OSTE(+) chips, respectively. 


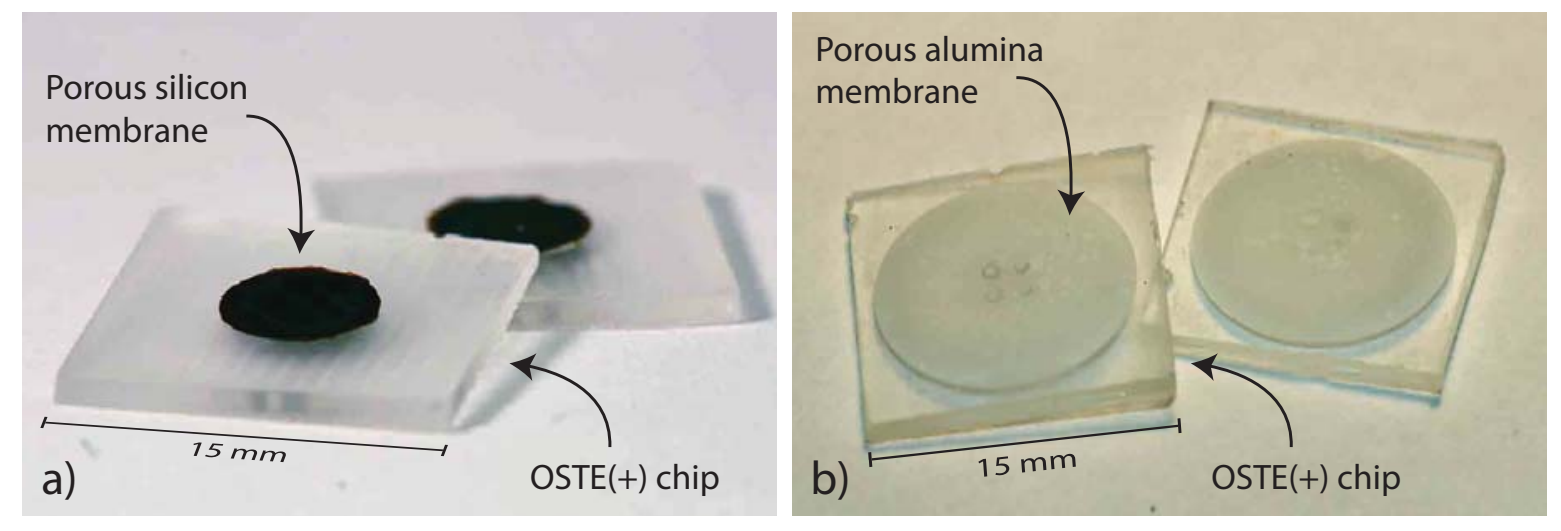

Figure 4. Photographs showing OSTE $(+)$ polymer chips with bonded: a) porous silicon membranes, and b) porous alumina membranes.

\section{Transfer bonding of porous alumina membranes to a microstructured silicon wafer using a thin $\operatorname{OSTE}(+)$ dry adhesive layer}

In this section, we demonstrate the batch transfer bonding of $13 \mathrm{~mm}$ diameter Whatman Anopore 200 por-Alu membranes onto a $100 \mathrm{~mm}$ diameter and $300 \mu \mathrm{m}$ thick microstructured silicon wafer (por-Alu/Si), using a lithographically patterned OSTE $(+)$ dry adhesive bonding layer. According to the supplier's specification, Anopore 200 membranes have a $60 \mu \mathrm{m}$ thickness, a $200 \mathrm{~nm}$ pore size, and $25 \%$ porosity.

The fabrication is illustrated in Figure 5. The OSTE $(+)$ polymer was prepared with a 1.5:1.0:0.5 ratio of thiol, allyl and epoxy, as described in Section 2. The components were mixed thoroughly and degassed in a vacuum desiccator. The silicon processing starts by a thermal growth of a $2.5 \mu \mathrm{m}$ thick $\mathrm{SiO}_{2}$ layer on an $\mathrm{Si}$ wafer, lithographic patterning of an $\mathrm{SiO}_{2}$ hard-mask on the wafer back side, and $\mathrm{KOH}$ etching of holes through the wafer (Figure $5 \mathrm{a}-\mathrm{b}$ ). After the etch, the front side openings of the through holes are still spanned by a thin oxide membrane, which was subsequently stripped by wet etching in a $50 \% \mathrm{HF}$-solution (Figure $5 \mathrm{c}$ ). Next, the wafer was partially diced, so that the dicing lines only penetrate $100 \mu \mathrm{m}$ into the wafer (Figure $5 \mathrm{~d}$ ). These groves serve as cleaving lines for separating the chips after bonding in the final step described below. The reason for cleaving the wafer instead of dicing is to avoid contaminating the nanoporous alumina membranes during a dicing process. The wafer with the cleaving lines was rinsed in DI water and coated with isocyanate silane, to improve the wetting of the $\operatorname{OSTE}(+)$ prepolymer of the silicon surface. This was done by immersing the wafer in 3\% w/w 3-isocyanatopropyl triethoxysilane (IPTES) dissolved in toluene for 20 minutes. After coating, the wafer was washed thoroughly with toluene, and oven baked at $110{ }^{\circ} \mathrm{C}$ for 10 minutes (Figure 5 e).

Dicing tape was attached to the backside of the patterned wafer before mounting the wafer on the spinner chuck (Figure $5 \mathrm{f}$ ). This was done to allow vacuum chuck fixation of the through etched wafer during spin coating. The OSTE $(+)$ polymer was spun to a thickness of $10 \mu \mathrm{m}$ at $3000 \mathrm{rpm}$ for $60 \mathrm{~s}$ and immediately flood exposed to UV 


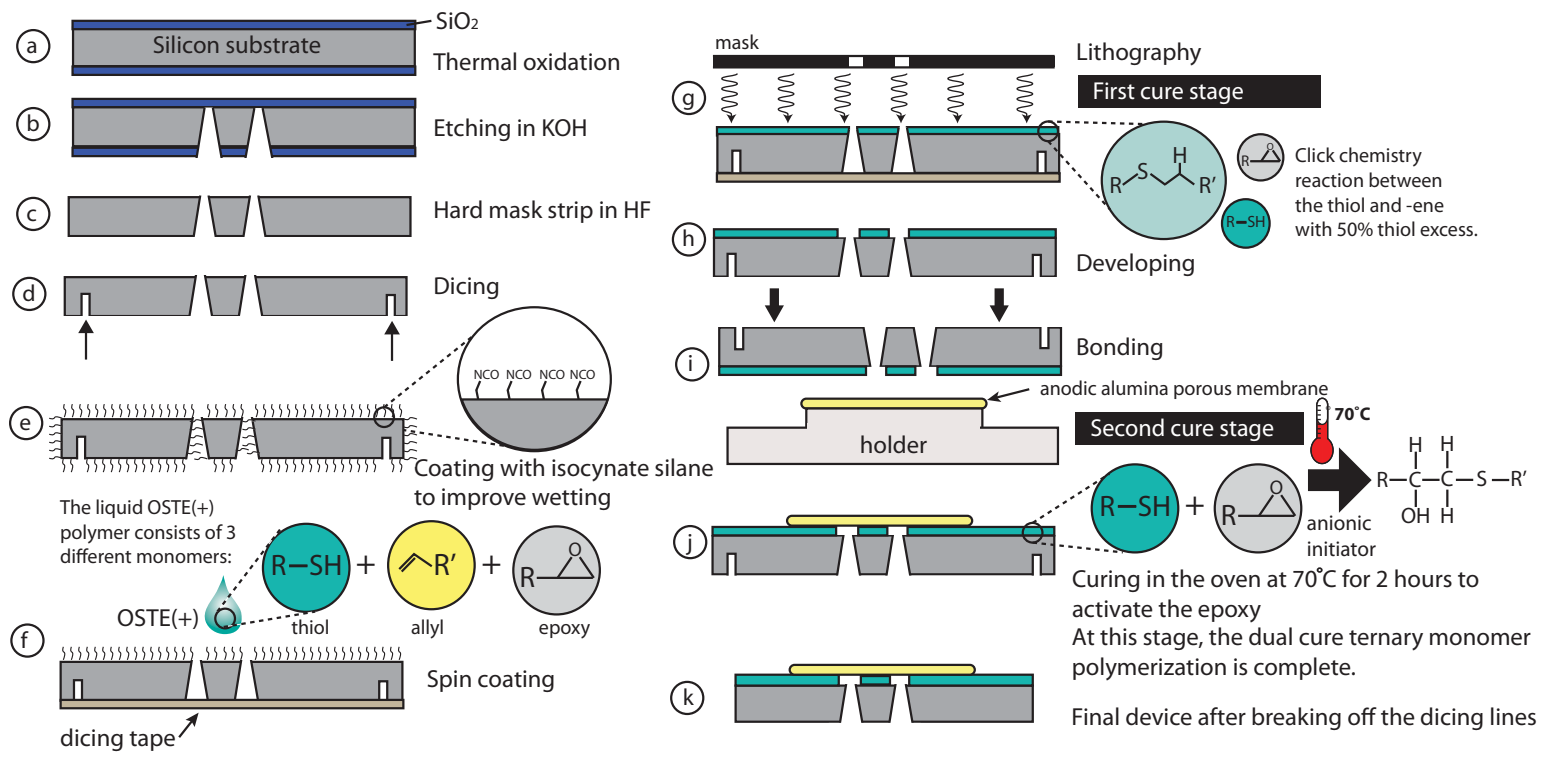

Figure 5. The wafer-scale fabrication process for the manufacturing of silicon chips with porous silicon membranes dry bonded using a $10 \mu \mathrm{m}$ thick OSTE $(+)$ bonding layer.

for $3 \mathrm{~s}$ at $20 \mathrm{~mW} / \mathrm{cm}^{2}$ in a Karl Suss MA6 mask aligner, to reduce flow of the OSTE $(+)$ into the silicon through-holes etched previously in $\mathrm{KOH}$. A mask defining the bonding area was then aligned to the wafer using proximity alignment mode, and exposed to UV for $2 \times 9 \mathrm{~s}$, with $10 \mathrm{~s}$ waiting time, to avoid raising the temperature and accelerating the second stage cure by the heating of the UV lamp (Figure $5 \mathrm{~g}$ ). The OSTE $(+)$ was then developed in toluene for $30 \mathrm{~s}$, as shown in Figure $5 \mathrm{~h}$ ).

After the first curing stage, the $\operatorname{OSTE}(+)$ surface contains unreacted thiol and epoxy groups, thus enabling the bonding to alumina. To enable batch transfer bonding of membranes, a plastic holder was created for the alignment of multiple por-Alu membranes in parallel to the target $100 \mathrm{~mm}$ Si wafer (Figure $5 \mathrm{i}$ ). The holder has $13 \mathrm{~mm}$ diameter circular protruding pillars, one for each membrane, as seen in the photograph in Figure 6 a). Alumina membranes were placed on each pillar of the holder, and then aligned and transferred in parallel to the silicon wafer covered with $\operatorname{OSTE}(+)$, by the guidance of alignment pins. The alignment pins provide an alignment accuracy of about $1 \mathrm{~mm}$. For more demanding applications, the alignment could be done in a mask aligner.

After the transfer, the bonded membranes were lightly pressed to the Si wafer, prior to the second curing stage in an oven at $70^{\circ} \mathrm{C}$ for 2 hours, to complete the polymerization (Figure $5 \mathrm{j}$ ). Finally, the chips were cleaved to size, using the pre-cut cleaving lines (Figure $5 \mathrm{k}$ ). Figure $6 \mathrm{~b}$ ) shows the final wafer with bonded por-Alu membranes, and the insets show close-up views of the front and back of a single por-Alu/Si chip. 

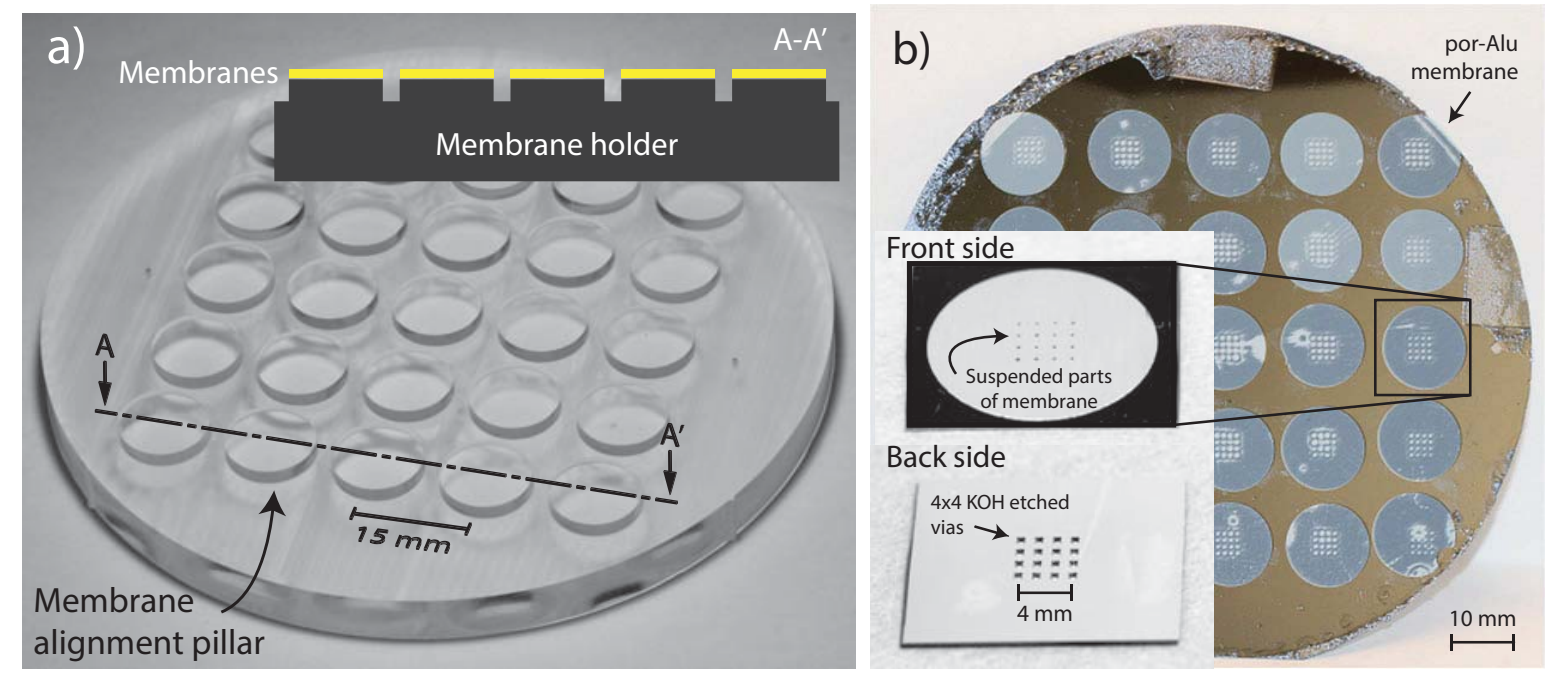

Figure 6. a) A photograph of the holder used to align and transfer 25 porous alumina membranes in parallel to a patterned silicon target wafer. The ruler scale is in $\mathrm{cm}$. The inset shows a schematic cross-section of the holder with aligned membranes. b) A photograph of the final wafer with transfer bonded porous alumina membranes. The insets show the front and the back of a single chip with a bonded porous alumina membrane. On the front side we see the $4 \times 4$ suspended areas of the membrane, and on the back side we see the corresponding $\mathrm{KOH}$ etched vias.

\section{Characterization}

To evaluate the membrane transfer bonding, two experiments were performed: First, the flow resistance of the bonded membranes was evaluated, to determine if any clogging had occurred during bonding. Second, the burst pressure for bonded membranes was determined, by slowly increasing the applied pressure until membrane delamination occurred at the $\operatorname{OSTE}(+)$ bond interface. Figure 7 shows a schematic of the testing setup.

To make a relevant comparison between the two presented bonding methods, we chose to use the more robust por-Alu membranes for testing both. OSTE $(+)$ polymer chips (por-Alu/OSTE $(+)$ ) and Si chips (por-Alu/Si), with bonded por-Alu membranes were prepared, as described in sections 3 and 4 . The por-Alu/OSTE $(+)$ chips contained 4 vias, each with a diameter of $800 \mu \mathrm{m}$, for a total membrane flow area of $0.5 \mathrm{~mm}^{2}$. The por-Alu/Si chips contained 16 vias with a square orifice of $64 \mu \mathrm{m}$ side length, and a total membrane flow area of approximately $0.1 \mathrm{~mm}^{2}$. The flow areas of the Si chips were larger than the orifice area, since the bond polymer leaks somewhat into the orifices after spinning and before flood exposure. This effect could be eliminated by modifying the process scheme in such a way that the oxide membrane left by the hard mask is not removed until after the bonding.

The flow characteristics were determined by pressuring deionized water through chips with bonded por-Alu membranes, while recording the pressure drop over the membranes and the resulting liquid flow. The chips were mounted in the flow setup 


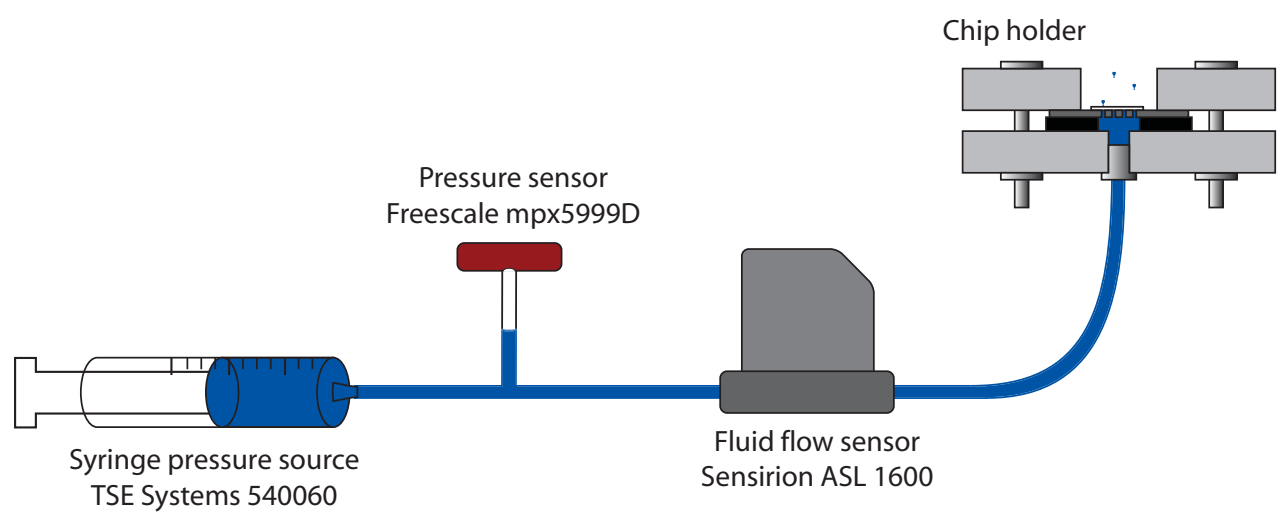

Figure 7. A schematic of the membrane flow testing setup. A syringe pump (TSE systems model 540060) was used as a liquid pressure source. A water filled hose connects the pressure source to a gas pressure sensor (Freescale mpx5999D), through a water pillar into an air filled cavity, measuring the differential air pressure resulting from the applied liquid pressure. The pressure driven flow through the tubing to the chip holder was gauged with a flow sensor (Sensirion ASL1600), and corresponds to the amount emitted through the openings of the porous membrane bonded to the chip. The chips were mounted in such a way that the bond interface was exposed to the applied pressure drop.

Table 1. A summary of the experimental conditions: substrate type, membrane type, number of vias, and total open flow area, as well as of the obtained results: normalized flow conductance, and burst pressure.

\begin{tabular}{|c|c|c|c|c|c|c|}
\hline Substrate & $\begin{array}{l}\text { Porous } \\
\text { alumina } \\
\text { membrane type }\end{array}$ & \# vias & $\begin{array}{l}\text { Via } \\
\text { diam. } \\
{[\mu \mathrm{m}]}\end{array}$ & $\begin{array}{l}\text { Flow } \\
\text { area } \\
{\left[\mathrm{mm}^{2}\right]}\end{array}$ & $\begin{array}{l}\text { Normalized } \\
\text { flow conductance } \\
{\left[\frac{\mu l}{\mathrm{~min} \cdot \mathrm{kPa} \cdot \mathrm{mm}^{2}}\right]}\end{array}$ & $\begin{array}{l}\text { Burst } \\
\text { pressure } \\
{[\mathrm{kPa}]}\end{array}$ \\
\hline $\operatorname{OSTE}(+)$ & Anopore 100 & 4 & 800 & 0.5 & 1.3 & 520 \\
\hline $\mathrm{Si}$ & Anopore 200 & 16 & 64 & $0.1^{\mathrm{a}}$ & 1.8 & 750 \\
\hline Reference & Anopore 100 & 1 & 650 & 0.3 & 1.5 & \\
\hline Reference & Anopore 200 & 1 & 480 & 0.2 & 1.3 & \\
\hline
\end{tabular}

${ }^{a}$ Larger than via area, due to flow of bond polymer before UV exposure.

with the bond interface exposed to the applied pressure. For the flow measurements, the flow was driven by a constant flow source (syringe pump), while both the pressure drop over the membranes and the resulting liquid flow were recorded as a function of time. These results were compared to the flow through reference samples consisting of untreated por-Alu membranes clamped with PDMS gaskets to flat aluminum plates with either 480 or $650 \mu \mathrm{m}$ diameter drilled aperture. Table 1 summarizes the conditions and results of the experiments.

To verify that the transfer bonding does not increase the flow resistance of the 


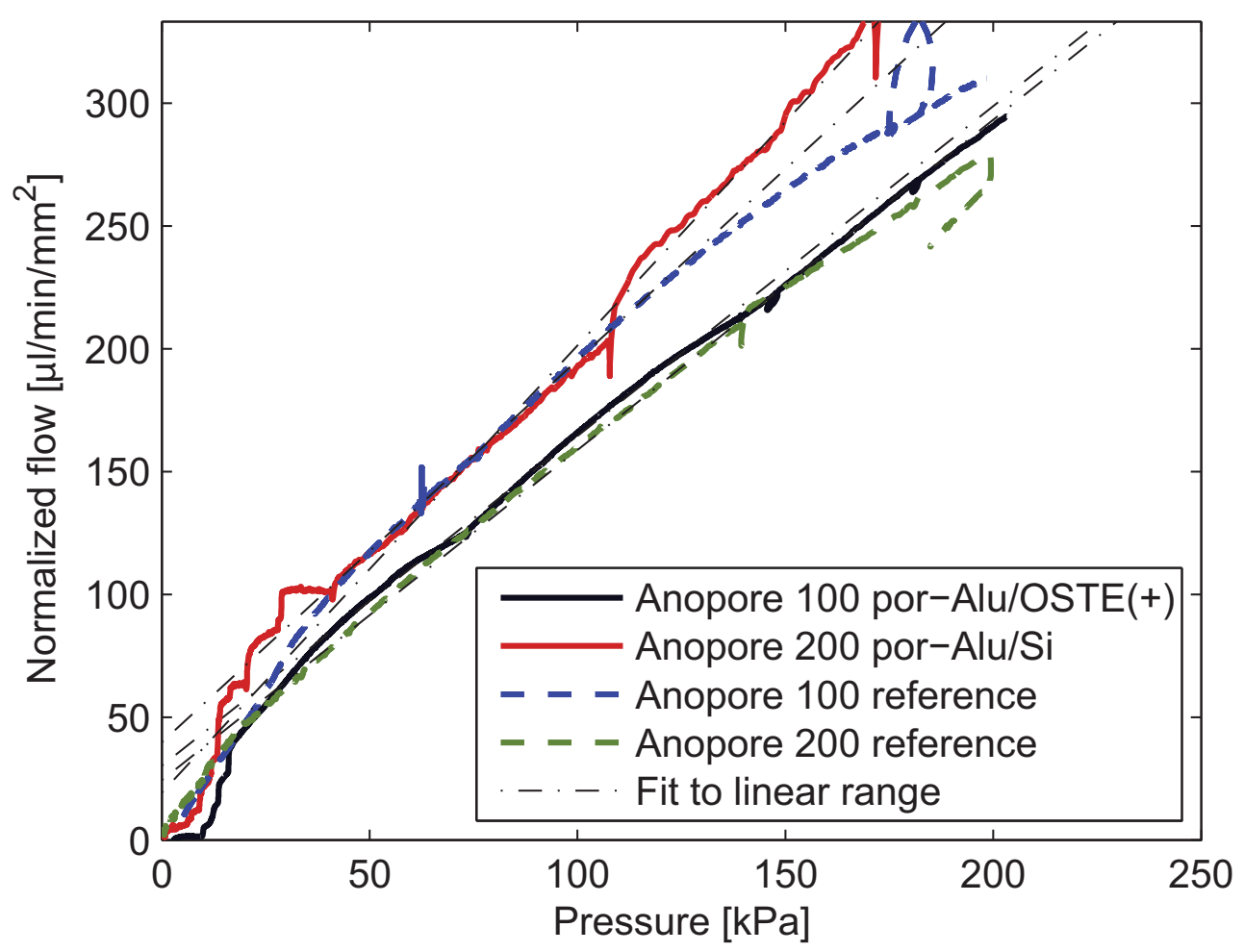

Figure 8. The membrane flow normalized by the flow area, as function of the pressure drop over the membranes.

membranes, e.g. by clogging, the measured membrane flows, normalized by their corresponding flow areas, are plotted as functions of applied pressure in Figure 8. Extracted values of the normalized flow conductance of bonded membranes corresponds well with that of the clamped reference membranes, as well as with that specified by the membrane producer $\left(1.2\right.$ and $1.5 \mu \mathrm{l} /\left(\mathrm{min} \cdot \mathrm{kPa} \cdot \mathrm{mm}^{2}\right)$, for Anopore 100 and 200, respectively), indicating that no clogging has occurred during membrane transfer and bonding.

For the burst pressure measurements, pressure was supplied by compressed air, which in turn pressurized a water filled tube that connected the pressure source to the chips. The pressure was slowly increased until the membranes delaminated from the substrate. Figure 9 shows the results. The membrane bonded to an OSTE $(+)$ chip delaminated at $520 \mathrm{kPa}$, and the membrane bonded to a silicon chip delaminated at the por-Alu/OSTE $(+)$ interface at $750 \mathrm{kPa}$. When compared to the pressures of 100-200 kPa applied in normal operations of microfluidic devices, the obtained burst pressures demonstrate the utility of the new bonding methods. 


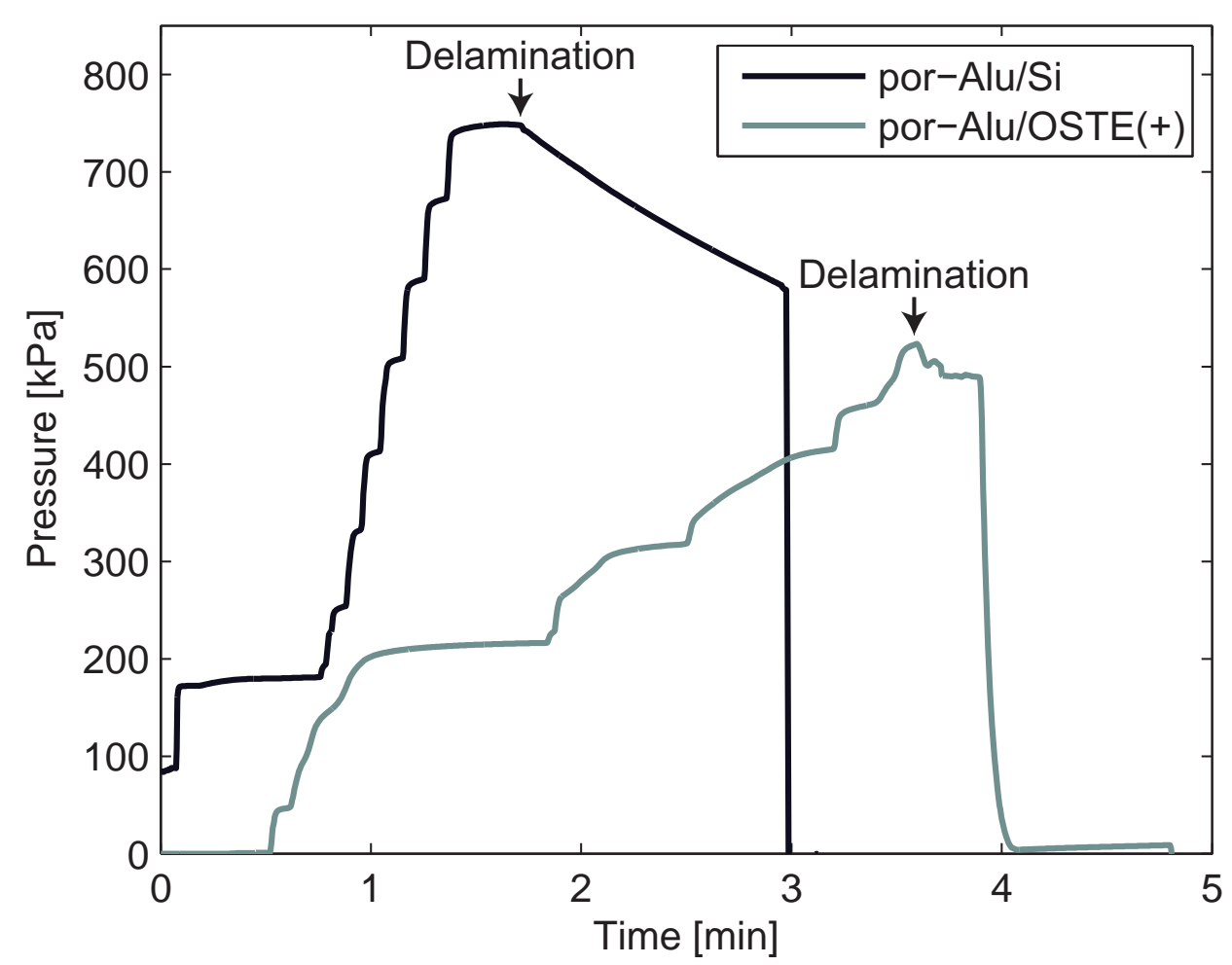

Figure 9. Burst pressure measurements of chips fabricated with the two integration schemes. The membrane bonded to an OSTE $(+)$ chip delaminated at $520 \mathrm{kPa}$, and the membrane bonded to a silicon chip delaminated at $750 \mathrm{kPa}$.

\section{Conclusions}

We have demonstrated two simple and robust ways to transfer inorganic nanoporous membranes to microfluidic devices. The transfer is enabled by the development of a new polymer formulation, OSTE $(+)$, which features a dual-cure polymerization specifically designed for bonding. We have described the two stage curing mechanism, and illustrated the usefulness of the methods by fabrication and characterization of simple microfluidic test chips. We evaluated the membrane bond strength, and confirmed that the bonding does not affect the flow properties of the membranes. The fact that the fabrication is carried out at the wafer-level leads to significant advantages, such as a reduced back-end process time and facile heterogeneous integration of microfluidics with CMOS electronics or MEMS structures.

\section{Acknowledgments}

This work was partially supported by the European Commission through the seventh framework project FP7-ICT-POSITIVE (grant agreement 257401) and the ERC starting grant ERC-M\&Ms (grant agreement 277879). 


\section{References}

[1] Christopher C. Striemer, Thomas R. Gaborski, James L. McGrath, and Philippe M. Fauchet. Charge- and size-based separation of macromolecules using ultrathin silicon membranes. Nature, 445(7129):749-753, February 2007.

[2] Hideki Masuda, Haruki Yamada, Masahiro Satoh, Hidetaka Asoh, Masashi Nakao, and Toshiaki Tamamura. Highly ordered nanochannel-array architecture in anodic alumina. Applied Physics Letters, 71(19):2770-2772, 1997.

[3] Marc G. Elgort, Mark G. Herrmann, Maria Erali, Jacob D. Durtschi, Karl V. Voelkerding, and Roger E. Smith. Extraction and Amplification of Genomic DNA from Human Blood on Nanoporous Aluminum Oxide Membranes. Clinical Chemistry, 50(10):1817-1819, October 2004.

[4] Grigori Sigalov, Jeffrey Comer, Gregory Timp, and Aleksei Aksimentiev. Detection of DNA Sequences Using an Alternating Electric Field in a Nanopore Capacitor. Nano Lett., 8(1):5663, December 2007.

[5] E. Anglin, L. Cheng, W. Freeman, and M. Sailor. Porous silicon in drug delivery devices and materials. Advanced Drug Delivery Reviews, 60(11):1266-1277, August 2008.

[6] Victor S. Y. Lin, Kianoush Motesharei, Keiki-Pua S. Dancil, Michael J. Sailor, and M. Reza Ghadiri. A Porous Silicon-Based Optical Interferometric Biosensor. Science, 278(5339):840843, October 1997.

[7] Sara D. Alvarez, Chang-Peng Li, Casey E. Chiang, Ivan K. Schuller, and Michael J. Sailor. A Label-Free Porous Alumina Interferometric Immunosensor. ACS Nano, 3(10):3301-3307, August 2009.

[8] D. J. Gargas, O. Muresan, D. J. Sirbuly, and S. K. Buratto. Micropatterned Porous-Silicon Bragg Mirrors by Dry-Removal Soft Lithography. Adv. Mater., 18(23):3164-3168, 2006.

[9] Guoguang Rong, Judson D. Ryckman, Raymond L. Mernaugh, and Sharon M. Weiss. Label-free porous silicon membrane waveguide for DNA sensing. Applied Physics Letters, 93(16):161109+, 2008.

[10] Andrew Carlson, Audrey M. Bowen, Yonggang Huang, Ralph G. Nuzzo, and John A. Rogers. Transfer Printing Techniques for Materials Assembly and Micro/Nanodevice Fabrication. Adv. Mater., 24(39):5284-5318, 2012.

[11] R. W. Tjerkstra, G. E. Gardeniers, J. J. Kelly, and A. van den Berg. Multi-walled microchannels: free-standing porous silicon membranesfor use in TAS. Microelectromechanical Systems, Journal of, 9(4):495-501, December 2000.

[12] Naxing Xu, Yuehe Lin, Steven A. Hofstadler, Dean Matson, Charles J. Call, and Richard D. Smith. A Microfabricated Dialysis Device for Sample Cleanup in Electrospray Ionization Mass Spectrometry. Anal. Chem., 70(17):3553-3556, August 1998.

[13] Fan Xiang, Yuehe Lin, Jenny Wen, Dean W. Matson, and Richard D. Smith. An Integrated Microfabricated Device for Dual Microdialysis and On-Line ESI-Ion Trap Mass Spectrometry for Analysis of Complex Biological Samples. Anal. Chem., 71(8):1485-1490, March 1999.

[14] Lutz Riegger, Oliver Strohmeier, Bernd Faltin, Roland Zengerle, and Peter Koltay. Adhesive bonding of microfluidic chips: influence of process parameters. Journal of Micromechanics and Microengineering, 20(8):087003+, July 2010.

[15] F. Niklaus, G. Stemme, J. Q. Lu, and R. J. Gutmann. Adhesive wafer bonding. Journal of Applied Physics, 99(3):031101+, 2006.

[16] J. De Jong, R. G. H. Lammertink, and M. Wessling. Membranes and microfluidics: a review. Lab Chip, 6(9):1125-1139, 2006.

[17] Carl F. Carlborg, Tommy Haraldsson, Kim Oberg, Michael Malkoch, and Wouter van der Wijngaart. Beyond PDMS: off-stoichiometry thiol-ene (OSTE) based soft lithography for rapid prototyping of microfluidic devices. Lab Chip, 11(18):3136-3147, 2011.

[18] Hartmuth C. Kolb, M. G. Finn, and K. Barry Sharpless. Click Chemistry: Diverse Chemical Function from a Few Good Reactions. Angewandte Chemie International Edition, 40(11):2004- 
2021, 2001.

[19] N. Sandstrom, R. Z. Shafagh, C. F. Carlborg, T. Haraldsson, G. Stemme, and W. van der Wijngaart. One step integration of gold coated sensors with OSTE polymer cartridges by low temperature dry bonding. In 2011 16th International Solid-State Sensors, Actuators and Microsystems Conference, pages 2778-2781. IEEE, June 2011.

[20] Carl F. Carlborg, Marina Cretich, Tommy Haraldsson, Laura Sola, M. Bagnati, Marcella Chiari, and Wouter van der Wijngaart. Biosticker: Patterned microfluidic stickers for rapid integration with microarrays. In 15th International Conference on Miniaturized Systems for Chemistry and Life Sciences, pages 311-313, October 2011.

[21] Farizah Saharil, Kristinn B. Gylfason, Yitong Liu, Tommy Haraldsson, Paolo Bettotti, Neeraj Kumar, and Wouter van der Wijngaart. Dry transfer bonding of porous silicon membranes to OSTE(+) polymer microfluidic devices. In 2012 IEEE 25th International Conference on Micro Electro Mechanical Systems (MEMS), pages 232-234. IEEE, January 2012.

[22] C. J. Oton, M. Ghulinyan, Z. Gaburro, P. Bettotti, L. Pavesi, L. Pancheri, S. Gialanella, and N. E. Capuj. Scattering rings as a tool for birefringence measurements in porous silicon. Journal of Applied Physics, 94(10):6334-6340, 2003. 